\title{
Biometric changes after vitrectomy with silicone oil tamponade
}

\author{
Simin Hosseini ${ }^{1}$, Amir Faramarzi ${ }^{2}$, Siamak Moradian ${ }^{2,3}$ and Mehdi Yaseri ${ }^{4}$ \\ ${ }^{1}$ Department of Optometry, School of Rehabilitation Sciences, Shahid Beheshti University of Medical Sciences, Tehran, Iran \\ ${ }^{2}$ Ophthalmic Research Center, Shahid Beheshti University of Medical Sciences, Tehran, Iran \\ ${ }^{3}$ Ophthalmic Epidemiology Research Center, Shahid Beheshti University of Medical Sciences, Tehran, Iran \\ ${ }^{4}$ Department of Epidemiology and Biostatistics, Tehran University of Medical Sciences, Tehran, Iran
}

\begin{abstract}
Background: The Lenstar LS 900 (Haag-Streit AG, Koeniz, Switzerland) is an optical biometer, and its measurements are highly repeatable and precise in cataractous eyes. This study investigated changes in biometric parameters, including axial length (AL), anterior chamber depth (ACD), lens thickness (LT), vitreous cavity depth (VD), and central corneal thickness (CCT) before and after three-port 23-Gauge pars plana vitrectomy with silicone oil tamponade.

Methods: This was a prospective follow-up study. Patients who were scheduled for surgery underwent a detailed slit-lamp examination and objective cycloplegic refraction preoperatively. In eligible cases, the Lenstar LS 900 was used to measure biometric parameters. At the 1-month postoperative follow-up, we repeated the same assessments for the silicone oil (SO)-filled eyes. Data were analyzed to assess the significance of changes and to test the possible correlation of values between the two time points.

Results: Twenty-three patients with a mean \pm standard deviation age of $60 \pm 12$ years completed the study. Postoperatively, we found a significant increase in AL and a decrease in ACD and CCT (all $P<0.05$ ), with no significant changes in LT and VD. A significant correlation was found for ACD, CCT, and cylinder values between the two time points (all $P<0.05$ ). Postoperatively, the spherical and cylindrical components of refraction demonstrated a hyperopic shift, but did not change statistically significantly.

Conclusions: The Lenstar LS 900 underestimated the ACD and overestimated the AL in SO-filled eyes when comparing pre- and postoperative values, in phakic as well as pseudophakic eyes. In planning for cataract surgery in this group of patients, it is more reasonable to calculate IOL power based on the biometric data of the fellow eye, although this may not eliminate possible errors. Further studies with a larger sample size, longer follow-up, and robust study design are necessary to confirm our preliminary results.
\end{abstract}

\section{KEYWORDS}

biometry, corneal thickness, anterior chamber depth, lens thickness, vitreous cavity, axial length, Lenstar LS 900, silicone oil

\section{INTRODUCTION}

Pars plana vitrectomy with silicone oil (SO) tamponade for repairing complicated retinal detachment was first reported by Cibis in 1962 [1]. Cataract is a frequent long-term complication in SO-filled phakic eyes [2]. Contact of the SO with the posterior surface of the lens affects its metabolism, resulting in cataract [3].

Correspondence: Simin Hosseini, Department of Optometry, School of Rehabilitation Sciences, Shahid Beheshti University of Medical Sciences, Tehran, Iran. E-mail: opt.siminhosseini@gmail.com. ORCID iD: https://orcid.org/0000-0003-0182-4872

How to cite this article: Hosseini S, Faramarzi A, Moradian S, Yaseri M. Biometric changes after vitrectomy with silicone oil tamponade. Med Hypothesis Discov Innov Optom. 2021 Summer; 2(2): 56-62. https://doi.org/10.51329/mehdioptometry126

Received: 01 May 2021; Accepted: 08 October 2021

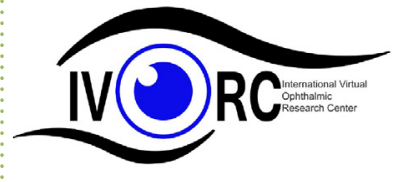

Copyright (C) Author(s). This is an open-access article distributed under the terms of the Creative Commons Attribution-NonCommercial 4.0 International License (http://creativecommons.org/licenses/by-nc/4.0/) which permits copy and redistribute the material just in noncommercial usages, provided the original work is properly cited. (c) (i) (s) 
Intraocular lens (IOL) power determination depends on three parameters: axial length (AL), anterior chamber depth (ACD), and average corneal power [2]. Imprecise measurement of AL, corneal power, and inaccurate estimation of postoperative ACD by optical biometry cause IOL power calculation error by $36 \%, 22 \%$, and $42 \%$, respectively [4].

The Lenstar LS 900 (Haag-Streit AG, Koeniz, Switzerland) is a new, non-contact optical biometer, introduced in 2008 [5]. This instrument uses the optical low coherence reflectometry technique [3, 5-9] with a broadband light source $(20-30 \mathrm{~nm})$ [7] to measure AL, ACD, central corneal thickness (CCT), lens thickness (LT) [3, $7-10]$, retinal thickness, keratometry readings $(\mathrm{K})[7,8,10]$, corneal diameter $(\mathrm{CD})[7]$, pupil size $[7,10]$, whiteto-white (WTW) distance (as horizontal iris width) $[3,10,11]$, and eccentricity of the pupil and visual optical line $[3,7]$. Optical biometry can facilitate a high degree of accuracy in IOL power estimation [12]. Compared to the IOLMaster, the reproducibility of the Lenstar LS 900 was excellent in healthy eyes [7], and its measurements were highly repeatable and precise in cataractous eyes $[3,13]$.

The precision of determining biometric parameters using Lenstar LS 900 in SO-filled eyes had to be sought. Hence, this study aimed to investigate changes in biometric parameters, including AL, ACD, LT, vitreous cavity depth (VD), and CCT using Lenstar LS 900 before and after deep vitrectomy with SO tamponade.

\section{METHODS}

This prospective follow-up study was conducted from July 2011 to September 2012 at the Labbafinejad Medical Center, Shahid Beheshti University of Medical Sciences, Tehran, Iran. The ethical committee of Shahid Beheshti University of Medical Sciences approved the study protocol, and a signed written informed consent was obtained from all participants before recruitment. The study adhered to the ethical principles of the tenets of the Declaration of Helsinki for human subjects.

We excluded patients with a history of any keratorefractive surgery, as such surgery could change the refractive index of the cornea [14] as compared to the untouched cornea. Patients who were unable to fixate on light for any reason, such as those with head tremors or corneal or lens opacities involving the visual axis, were also excluded. We excluded patients with keratoconus or any ectatic corneal diseases due to the presence of irregular astigmatism and the possibility of its change within a 1-month study duration. Eligible participants were recruited using the convenience sampling method during a 1-month study period.

Initially, from cases referred to the ophthalmology clinic at Labbafinejad Medical Center, patients who were scheduled for deep vitrectomy with SO tamponade underwent a thorough anterior and posterior segment slitlamp examination preoperatively, by a senior ophthalmologist trained in the retina subspecialty, to evaluate retinal, corneal, and lens pathologies. For 30 eyes in which it was deemed appropriate to proceed, the Lenstar LS 900 non-contact biometer (Haag-Streit AG, Koeniz, Switzerland) was used to measure biometric parameters, including AL, ACD, LT, CCT, and VD. These parameters were measured preoperatively, between 09:00 AM and 12:00 PM, by the same technician. According to manufacturer's instructions of the Lenstar LS 900, anesthetic eye drops were not administered before the evaluation. The measurements were performed five consecutive times, and the mean value was calculated and used for the final analysis. Furthermore, objective cycloplegic refraction was performed and subsequently subjectively refined.

Standard three-port 23-Gauge pars plana vitrectomy with SO tamponade was performed for all eyes, as described elsewhere [15]. The same surgeon, using similar surgical techniques and instruments, performed all surgeries. The viscosity, level of purification, and chemical composition of the different types of SO vary. The viscosity depends on the polymeric compound length and ranges from 1000 to 10000 centistokes (cs). Two types of highly purified polydimethylsiloxanes, low viscosity (1000 or $1300 \mathrm{cs)}$ ) and high viscosity (5000 or $5700 \mathrm{cs}$ ), are commonly used in clinical practice [16]. In this study, 5700 cs SO (Oxane 5700; Bausch \& Lomb, Germany) was injected in all patients.

At the 1-month postoperative follow-up, the same ophthalmologist repeated detailed slit-lamp examination for all included eyes. Patients who did not attend the postoperative follow-up, those with a hazy cornea, trauma, recurrent retinal detachment, or who were severely affected by the underlying disease were excluded from the final analysis. Thus, for 23 remaining eyes, the same technician repeated the measurement of biometric parameters using the Lenstar LS 900, as described above, and again performed objective cycloplegic refraction, which was then refined subjectively.

All analyses were performed using IBM SPSS Statistics for Windows (version 20.0; IBM Corp., Armonk, Chicago, IL, USA). Variables were expressed as mean \pm standard deviation (SD) or frequency and percentage. We used the Wilcoxon signed-rank test to compare pre-and postoperative values and the Mann-Whitney $U$ test to compare the phakic and pseudophakic subgroups. Pearson's product-moment correlation was performed to test 
the possible correlation between pre-and post-operative values. The level of significance was set at $P<0.05$.

\section{RESULTS}

Twenty-three eyes of 23 patients with a mean \pm SD age of $60 \pm 12$ years were included in the final analysis. Table 1 shows the baseline characteristics and clinical specifications of the study participants.

Table 2 shows the mean \pm SD of biometry parameters and refraction and their pre- and postoperative changes in all participants. Postoperatively, a significant increase in AL and a decrease in ACD and CCT (all $P<0.05$ ) were found. The changes in LT and VD, despite showing a postoperative increase in mean values, were not statistically significant $(P=0.779$ and $P=0.091$, respectively). Furthermore, only pre- and postoperative ACD $(\mathrm{r}=$ $0.913 ; P<0.05)$, CCT $(\mathrm{r}=0.770 ; P<0.01)$, and cylinder $(\mathrm{r}=0.662 ; P<0.05)$ values were significantly correlated (Table 2). Postoperatively, the spherical and cylindrical components of refraction demonstrated a hyperopic shift by $+1.28 \pm 5.6 \mathrm{D}$ and $+0.13 \pm 1.82 \mathrm{D}$, respectively; however, these changes were not statistically significant for either component $(P=0.075$ and $P=0.788$, respectively) (Table 2$)$.

Table 3 shows a comparison of biometry parameters pre-and postoperatively in the phakic and pseudophakic eye subgroups. Except for significantly deeper pre- and postoperative ACD in pseudophakic eyes (both $P<$ 0.05), other parameters did not differ significantly between the two subgroups (Table 3 ).

Comparing pre- versus postoperative parameters within each subgroup, a significant increase in AL in both subgroups (both $P<0.05$ ), and a significant decrease in the CCT of pseudophakic eyes $(P<0.05)$ was found postoperatively (Table 3).

Table 1. Baseline characteristics and clinical specifications of study participants

\begin{tabular}{|c|c|c|}
\hline Parameter & \multicolumn{2}{|l|}{ Value } \\
\hline Age, $(y)$, Mean \pm SD $/$ Median (Range) & \multicolumn{2}{|c|}{$60 \pm 12 / 60(36$ to 81$)$} \\
\hline Gender, n (\%), Male/Female & \multicolumn{2}{|c|}{$15(65.2) / 8(34.8)$} \\
\hline Eye, n (\%), OD/OS & \multicolumn{2}{|c|}{$14(60.9) / 9(39.1)$} \\
\hline \multirow[t]{5}{*}{ Pathology*, n (\%) } & Trauma & $3(13.1)$ \\
\hline & Diabetes Mellitus & $10(43.47)$ \\
\hline & Hypertension & $5(21.73)$ \\
\hline & Senile Cataract & $6(26.1)$ \\
\hline & Aphakic & $2(8.7)$ \\
\hline \multirow[t]{3}{*}{ Lens type, n (\%) } & Phakic & $10(43.5)$ \\
\hline & Pseudophakic & $11(47.8)$ \\
\hline & Aphakic & $2(8.7)$ \\
\hline
\end{tabular}

Abbreviations: y, years; SD, standard deviation; n, number; \%, percentage; OD, right eye; OS, left eye *As there were subjects with more than one pathology, the sum of the percentage from this variable exceeds $100 \%$.

Table 2. Biometric parameters and refraction pre- and post-vitrectomy with silicone oil tamponade in all participants

\begin{tabular}{|l|l|l|l|l|l|}
\hline Parameter & $\begin{array}{l}\text { Pre } \\
\text { Mean } \pm \text { SD }\end{array}$ & $\begin{array}{l}\text { Post } \\
\text { Mean } \pm \text { SD }\end{array}$ & Correlation & $\begin{array}{l}\text { Change (Post-Pre) } \\
\text { Mean } \pm \text { SD (95\% CI) }\end{array}$ & \multicolumn{1}{|l|}{-value } \\
\hline ACD $(\mathbf{m m})$ & $3.91 \pm 1.01$ & $3.64 \pm 0.95$ & $0.913^{*}$ & $-0.22 \pm 0.39(-0.01$ to 0.44$)$ & $\mathbf{0 . 0 4 8}$ \\
\hline LT $(\mathbf{m m})$ & $3.65 \pm 1.54$ & $3.79 \pm 1.46$ & 0.553 & $-0.39 \pm 1.28(-0.6$ to 1.38$)$ & 0.779 \\
\hline VD $(\mathbf{m m})$ & $16.09 \pm 2.12$ & $16.78 \pm 1.62$ & 0.475 & $1.21 \pm 2.1(-3.16$ to 0.73$)$ & 0.091 \\
\hline AL $(\mathbf{m m})$ & $23.01 \pm 1.98$ & $24.85 \pm 2.18$ & 0.364 & $1.82 \pm 2.49(3.02$ to -0.62$)$ & $\mathbf{0 . 0 0 2}$ \\
\hline CCT $(\boldsymbol{\mu m})$ & $539.0 \pm 34.0$ & $526.0 \pm 36.0$ & $0.770^{* *}$ & $-13.3 \pm 23.87(2.98$ to 23.63$)$ & $\mathbf{0 . 0 0 4}$ \\
\hline Sphere $(\mathbf{D})$ & $-0.28 \pm 2.84$ & $+2.62 \pm 4.98$ & 0.178 & $+1.28 \pm 5.6(-4.66$ to +2.11$)$ & 0.075 \\
\hline Cylinder $(\mathbf{D})$ & $-1.51 \pm 1.34$ & $-1.87 \pm 2.37$ & $0.662^{*}$ & $+0.13 \pm 1.82(-1.24$ to +0.97$)$ & 0.788 \\
\hline
\end{tabular}

Abbreviations: Pre, pre-vitrectomy with silicone oil tamponade; Post, post-vitrectomy with silicone oil tamponade; SD, standard deviation; ACD, anterior chamber depth; mm, millimeter; LT, lens thickness; VD, vitreous cavity depth; AL, axial length; CCT, central corneal thickness; $\mu \mathrm{m}$, micrometer; sphere, spherical component of refraction; cylinder, cylindrical component of refraction; D, diopter. ${ }^{*} P<0.05$; ${ }^{* *} P<0.01$; ${ }^{\ddagger}$ Based on Wilcoxon signed-rank test. Significant $P$-values are shown in bold. 
Table 3. The comparison of biometry parameters pre-and post-vitrectomy with silicone oil tamponade in the two subgroups of phakic and pseudophakic eyes

\begin{tabular}{|c|c|c|c|c|}
\hline Parameter & Assessment Time & $\begin{array}{l}\text { Phakic } \\
(\mathrm{n}=10 \text { eyes }) \\
\text { Mean } \pm \text { SD }\end{array}$ & $\begin{array}{l}\text { Pseudophakic } \\
\text { ( } \mathrm{n}=11 \text { eyes }) \\
\text { Mean } \pm \text { SD }\end{array}$ & $\begin{array}{l}P \text {-value }{ }^{*} \\
\text { (Comparison between subgroups) }\end{array}$ \\
\hline \multirow[t]{4}{*}{$\operatorname{ACD}(\mathbf{m m})$} & Preoperative & $3.32 \pm 0.39$ & $4.77 \pm 0.74$ & 0.001 \\
\hline & Postoperative & $3.01 \pm 0.31$ & $4.49 \pm 0.83$ & 0.003 \\
\hline & Change & $-0.24 \pm 0.46$ & $-0.19 \pm 0.31$ & 0.796 \\
\hline & $P$-value within group $\neq$ & 0.092 & 0.249 & \\
\hline \multirow[t]{4}{*}{$\mathbf{L T}(\mathbf{m m})$} & Preoperative & $4.38 \pm 0.24$ & $2.01 \pm 2.04$ & 0.164 \\
\hline & Postoperative & $4.47 \pm 0.30$ & $1.08 \pm 0.28$ & 0.036 \\
\hline & Change & $0.04 \pm 0.09$ & $-1.91 \pm 2.67$ & 0.106 \\
\hline & $P$-value within group $\neq$ & 0.249 & 0.180 & \\
\hline \multirow[t]{4}{*}{ VD (mm) } & Preoperative & $15.87 \pm 1.60$ & $16.47 \pm 3.09$ & 0.450 \\
\hline & Postoperative & $16.33 \pm 1.45$ & $18.56 \pm 0.95$ & 0.117 \\
\hline & Change & $0.54 \pm 0.73$ & $2.90 \pm 4.04$ & 0.699 \\
\hline & $P$-value within group $\neq$ & 0.225 & 0.180 & \\
\hline \multirow[t]{4}{*}{$\mathrm{AL}(\mathbf{m m})$} & Preoperative & $23.15 \pm 2.09$ & $22.91 \pm 1.99$ & 0.869 \\
\hline & Postoperative & $24.48 \pm 2.02$ & $25.15 \pm 2.56$ & 0.439 \\
\hline & Change & $1.23 \pm 1.37$ & $2.24 \pm 3.06$ & 0.710 \\
\hline & $P$-value within group $\neq$ & 0.036 & 0.017 & \\
\hline \multirow[t]{4}{*}{$\mathrm{CCT}(\mu \mathrm{m})$} & Preoperative & $541 \pm 27$ & $531 \pm 33$ & 0.459 \\
\hline & Postoperative & $524 \pm 31$ & $521 \pm 37$ & 0.888 \\
\hline & Change & $-17.6 \pm 33.42$ & $-10.09 \pm 14.38$ & 0.916 \\
\hline & $P$-value within group $\neq$ & 0.126 & 0.029 & \\
\hline
\end{tabular}

Abbreviations: Preoperative, pre-vitrectomy with silicone oil tamponade; Postoperative, post-vitrectomy with silicone oil tamponade; SD, standard deviation; n, number; ACD, anterior chamber depth; mm, millimeter; LT, lens thickness; VD, vitreous cavity depth; AL, axial length; CCT, central corneal thickness; $\mu \mathrm{m}$, micrometer. $P$-values less than 0.05 are shown in bold. ${ }^{*}$ Based on the Mann-Whitney test; ${ }^{\ddagger}$ Based on the Wilcoxon signed-rank test.

\section{DISCUSSION}

This study investigated changes in biometric parameters (AL, ACD, LT, VD, and CCT) before and after deep vitrectomy with SO tamponade. The results revealed a significant increase in AL and a decrease in the ACD and CCT. No significant changes were found in LT and VD, despite a postoperative increase in mean values. Furthermore, a significant correlation was found between the pre- and postoperative ACD, CCT, and cylinder values. Postoperatively, the spherical and cylindrical components of refraction had a hyperopic shift, yet these changes were not significant. Except for significantly deeper pre- and postoperative ACD in pseudophakic versus phakic eyes, other parameters did not differ significantly between them. This might be due to anterior shifting of the crystalline lens in phakic eyes postoperatively, as we detected a $0.24-\mathrm{mm}$ reduction in mean ACD in this subgroup, but the change was not significant. A significant increase was found postoperatively in the AL of both pseudophakic and phakic eyes, and a significant decrease was found in the CCT of pseudophakic eyes postoperatively.

Use of SO in phakic eyes accelerates cataract formation. It also causes errors in IOL power calculations for a number of reasons, such as incomplete filling of the vitreous chamber with $\mathrm{SO}$, a partially removed vitreous base, biometry performed in a supine position, implantation of a sulcus IOL, presence of edema or detachment in the macula, and use of outdated formulas in IOL power calculation [17]. In SO-filled eyes, the accuracy of AL measurements is affected by the altered refractive index of the eye. The optical path length of the posterior segment in the SO-filled eye is altered; therefore, an average $+6 \mathrm{D}$ shift in the refraction of phakic SO-filled eyes is plausible $[4,17]$. Hence, the interference of SO with the assessment of ocular biometric parameters must be taken into account in the calculation of IOL power for SO-filled eyes during treatment planning.

In the current study, we employed the Lenstar LS 900 to measure biometric parameters preoperatively and 1 month after three-port 23-Gauge pars plana vitrectomy with SO tamponade. The reproducibility and accuracy 
of the Lenstar LS 900 have been verified previously by Rabsilber et al. [5], Shammas et al. [18], and Bjeloš Rončević et al. [19] in cataractous eyes, by Shen et al. [20] and Zhao et al. [21] in myopic eyes, as well as in the normal eyes of adults and school-aged children by Schulle et al. [22] and Şahin et al. [23], respectively. Furthermore, Kunavisarut et al. [24], Roessler et al. [25], and Parravano et al. [26] proved the high precision and repeatability of the IOLMaster, an optical biometer similar to the Lenstar LS 900, in measuring the AL of SO-filled eyes. In contrast, we found that ACD was underestimated by the Lenstar LS 900 in SO-filled eyes. Additionally, it overestimated AL in both phakic and pseudophakic SO-filled eyes.

Rohrer et al. [6] compared biometric parameters, including AL, ACD, CCT, corneal radius, keratometry of flat and steep radii, and the axis of the flattest radius in 144 cataractous, pseudophakic, aphakic, SO-filled, or normal eyes measured using the Lenstar LS 900, IOLMaster (Carl Zeiss Meditec, Jena, Germany), and Pach (Haag-Streit AG). They found a good correlation between Lenstar LS 900 measurements in all included eyes, with all instruments [6]. The current study found a significant correlation between pre- and postoperative ACD, CCT, and cylinder values. However, other parameters, namely LT, VD, AL, and the spherical component of refraction, showed no significant correlation, despite having a positive $\mathrm{r}$ value.

Liu et al. [27] investigated the clinical results of ultrasound biometry in SO-filled eyes. They reported that the ultrasound biometer allows accurate and simple measurements in such eyes. The AL measured by ultrasound biometry did not differ significantly before and after SO removal, but the mean VD decreased from $26.57 \mathrm{~mm}$ to $17.90 \mathrm{~mm}$ [27]. In contrast, using the Lenstar LS 900 overestimated AL in SO-filled eyes, and this remained true when values for both phakic and pseudophakic eyes were compared between the two time points. This discrepancy between our study and that of Liu et al. [27] may stem from the fact that these two studies employed different types of devices. However, further comparative studies are needed to confirm our findings.

Roessler et al. [25] assessed the precision of AL measurements in 26 eyes with SO, using the IOLMaster. AL was measured on the day before and 6 weeks after SO removal. The results revealed acceptable accuracy for AL measured using the IOLMaster [25]. However, although we employed the Lenstar LS 900, which is an optical biometer similar to the IOLMaster, we found that AL was overestimated after SO injection as compared with preoperative biometry. The difference might be due to the timing of measurements, as well as the difference in biometry devices used in these two studies. However, our rationale should be verified through further comparative studies.

Wang et al. [28] measured AL and ACD before and after SO removal using A-scan ultrasonography and IOLMaster in 67 eyes. After removal of SO, AL values were reduced significantly when measured using the IOLMaster. The AL values obtained by the two devices showed good agreement before and after SO removal; however, the AL measured by the IOLMaster was more accurate. Both pre- and post-SO removal ACD values were significantly lower when measured by the A-scan than when measured by the IOLMaster [28]. Given that we did not compare our measurements with values obtained by either A-scan ultrasonography or the IOLMaster, it is difficult to ascertain the accuracy of the Lenstar LS 900 in SO-filled eyes. However, compared with pre-injection values, we found that $\mathrm{AL}$ was overestimated in SO-filled eyes, which could interfere with an accurate estimation of IOL power in these eyes. Further studies are needed to clarify this matter.

Liu and $\mathrm{Li}$ [29] measured ocular biometric parameters (AL, ACD, and LT) before and after vitrectomy with SO injection in phakic eyes using an IOLMaster 700 [29]. Similar to the current study, the postoperative mean $\mathrm{AL}$ and mean ACD values increased and decreased, respectively. This might be due to the measurement error of AL following SO tamponade. We found deeper pre- and postoperative ACD in pseudophakic versus phakic eyes, as detected by Liu and $\mathrm{Li}$ [29] in their participants with phakic eyes. These similar results might be due to the postoperative anterior shift of the crystalline lens in phakic eyes. Robust verification could be achieved by comparing these two optical biometers in two groups of well-matched SO-filled eyes.

Kulikov et al. [30] measured the AL of SO-filled and control eyes using the IOLMaster and the Lenstar LS 900. For a better interpretation of the data, the eyes were subdivided into those with AL $>23.63 \mathrm{~mm}$ and those with $\mathrm{AL}<23.63 \mathrm{~mm}$. Both biometers significantly overestimated the $\mathrm{AL}$ of SO-filled eyes with a longer AL, although this error was smaller with the Lenstar LS 900 than with the IOLMaster. Biometry was reported to be more accurate in SO-filled eyes with a shorter AL [30]. Likewise, we found that the Lenstar LS 900 overestimated AL in SO-filled eyes when we compared pre- and postoperative values in both phakic and pseudophakic eyes.

In the current study, ACD was significantly underestimated, and AL was significantly overestimated in SOfilled eyes by the Lenstar LS 900. Inaccurate measurement of AL and incorrect estimation of postoperative ACD by optical biometry cause IOL power calculation error by $36 \%$ and $42 \%$, respectively [4]. Therefore, in planning for cataract surgery in phakic SO-filled eyes, other alternatives for IOL power calculation, such as calculating IOL power before $\mathrm{SO}$ injection, using the biometric data of the fellow eye, or performing biometry after $\mathrm{SO}$ removal 
should be considered. Likewise, the Lenstar LS 900 underestimated CCT in SO-filled eyes. The CCT was significantly positively correlated with AL in healthy eyes [31]. Therefore, in SO-filled eyes, Lenstar 900 may cause IOL power calculation error and IOL surprise postoperatively. In contrast, Piasecka et al. [32] employed the Lenstar LS 900 for biometry in SO-filled eyes for IOL power calculation in combined SO removal and phacoemulsification surgery, and compared the results with those of a control group. The postoperative refractive outcome was as accurate as that in the non-vitrectomized control eyes. They concluded that the Lenstar LS 900 enables accurate IOL power calculations in SO-filled eyes [32].

The present study sought to detect changes in biometric parameters pre-and postoperatively, which offer advantages in comparison and provide more reliable outcomes. However, it had the following limitations: it lacked a control group, had a small sample size, a short follow-up period, and lacked investigation of age or sex subgroups. Moreover, in this study, we did not subdivide participants based on the severity of the underlying refractive error or the AL, which could be helpful in IOL power calculation of SO-filled eyes. Future studies addressing these limitations could establish more conclusive results and may provide a solution to the under- and overestimations of IOL power in this specific group of patients.

\section{CONCLUSIONS}

The Lenstar LS 900 underestimated the ACD and overestimated the AL of SO-filled eyes, based on comparison of pre- and postoperative values in both phakic and pseudophakic eyes. When planning for cataract surgery in this group of patients, it is more reasonable to calculate IOL power before SO injection. If this is not feasible, the biometric data of the fellow eye or from biometry performed after SO removal are alternatives. However, these measures may not guarantee total elimination of possible errors. Further studies with a larger sample size, longer follow-up, and robust study design are necessary to confirm our preliminary results.

\section{ETHICAL DECLARATIONS}

Ethical approval: The ethics committee of Shahid Beheshti University of Medical Sciences approved the study protocol and a signed written informed consent was obtained from all participants before recruitment. The study adhered to the ethical principles of the tenets of the Declaration of Helsinki for human subjects.

Conflict of interests: None

\section{FUNDING}

\section{None.}

\section{ACIKNOWLED GMENTS}

This paper is derived from the final report of a Master's dissertation in optometry and received ethical approval from Shahid Beheshti University of Medical Sciences, Tehran, Iran. We express our gratitude and appreciation to the Vice-Chancellor for Research at the Shahid Beheshti University of Medical Sciences. Additionally, we thank all the colleagues and participants who helped us conduct and complete this study.

\section{REFERENCES}

1. Rezaei KA, Abrams GW (2005). 'The history of retinal detachment surgery'. In Primary Retinal Detachment 2005 (pp. 1-24). Springer, Berlin, Heidelberg. Link

2. Murray DC, Durrani OM, Good P, Benson MT, Kirkby GR. Biometry of the silicone oil-filled eye: II. Eye. 2002;16(6):72730. doi: 10.1038/sj.eye.6700176 pmid: 12439667

3. Buckhurst PJ, Wolffsohn JS, Shah S, Naroo SA, Davies LN, Berrow EJ. A new optical low coherence reflectometry device for ocular biometry in cataract patients. Br J Ophthalmol. 2009;93(7):949-53. doi: 10.1136/bjo.2008.156554 pmid: 19380310

4. Olsen T. Calculation of intraocular lens power: a review. Acta Ophthalmol Scand. 2007;85(5):472-85. doi: 10.1111/j.16000420.2007.00879.x pmid: 17403024

5. Rabsilber TM, Jepsen C, Auffarth GU, Holzer MP. Intraocular lens power calculation: Clinical comparison of 2 optical biometry devices. J Cataract Refract Surg. 2010;36(2):230-4. doi: 10.1016/j.jcrs.2009.09.016 pmid: 20152602

6. Rohrer K, Frueh BE, Wälti R, Clemetson IA, Tappeiner C, Goldblum D. Comparison and Evaluation of Ocular Biometry Using a New Noncontact Optical Low-Coherence Reflectometer. Ophthalmology. 2009;116(11):2087-92. doi: 10.1016/j. ophtha.2009.04.019 pmid: 19744720

7. Cruysberg LPJ, Doors M, Verbakel F, Berendschot TTJM, De Brabander J, Nuijts RMMA. Evaluation of the Lenstar LS 900 non-contact biometer. Br J Ophthalmol. 2009;94(1):106-10. doi: 10.1136/bjo.2009.161729 pmid: 19692383

8. Hoffer KJ, Shammas JH, Savini G. Comparison of 2 laser instruments for measuring axial length. J Cataract Refract 
Surg. 2010;36(4):644-8. doi: 10.1016/j.jcrs.2009.11.007. Erratum in: J Cataract Refract Surg. 2010 Jun;36(6):1066 pmid: 20362858

9. Tappeiner C, Rohrer K, Frueh BE, Waelti R, Goldblum D. Clinical comparison of biometry using the non-contact optical low coherence reflectometer (Lenstar LS 900) and contact ultrasound biometer (Tomey AL-3000) in cataract eyes. Br J Ophthalmol. 2010;94(5):666-7. doi: 10.1136/bjo.2009.167700 pmid: 20447976

10. Bjeloš Rončević M, Bušić M, Čima I, Kuzmanović Elabjer B, Bosnar D, Miletić D. Comparison of optical low-coherence reflectometry and applanation ultrasound biometry on intraocular lens power calculation. Graefes Arch Clin Exp Ophthalmol. 2011;249(1):69-75. doi: 10.1007/s00417-010-1509-4 pmid: 20853004

11. O’Donnell C, Hartwig A, Radhakrishnan H. Correlations between refractive error and biometric parameters in human eyes using the LenStar 900. Cont Lens Anterior Eye. 2011;34(1):26-31. doi: 10.1016/j.clae.2010.10.006 pmid: 21081280

12. Manvikar SR, Allen D, Steel DHW. Optical biometry in combined phacovitrectomy. J Cataract Refract Surg. 2009;35(1):649. doi: 10.1016/j.jcrs.2008.09.020 pmid: 19101426

13. Salouti R, Nowroozzadeh MH, Zamani M, Ghoreyshi M, Salouti R. Comparison of the ultrasonographic method with 2 partial coherence interferometry methods for intraocular lens power calculation. Optometry. 2011;82(3):140-7. doi: 10.1016/j. optm.2010.07.025 pmid: 20933477

14. Borasio E, Stevens J, Smith GT. Estimation of true corneal power after keratorefractive surgery in eyes requiring cataract surgery: BESSt formula. J Cataract Refract Surg. 2006;32(12):2004-14. doi: 10.1016/j.jcrs.2006.08.037 pmid: 17137976

15. Nourinia R, Borna F, Rahimi A, Jabbarpoor Bonyadi Mohammad H, Amizadeh Y, Daneshtalab A, et al. Repeated Injection of Methotrexate into Silicone Oil-Filled Eyes for Grade C Proliferative Vitreoretinopathy: A Pilot Study. Ophthalmologica. 2019;242(2):113-7. doi: 10.1159/000500271 pmid: 31163427

16. Ratanapakorn T, Thongmee W, Meethongkam K, Sinawat S, Sanguansak T, Bhoomibunchoo C, et al. Emulsification of Different Viscosity Silicone Oil in Complicated Retinal Detachment Surgery: A Randomized Double-Blinded Clinical Trial. Clin Ophthalmol. 2020;14:359-67. doi: 10.2147/OPTH.S242804. pmid: 32103882

17. Kanclerz P, Grzybowski A. Accuracy of Intraocular Lens Power Calculation in Eyes Filled with Silicone Oil. Semin Ophthalmol. 2019;34(5):392-7. doi: 10.1080/08820538.2019.1636097 pmid: 31257972

18. Shammas HJ, Hoffer KJ. Repeatability and Reproducibility of Biometry and Keratometry Measurements Using a Noncontact Optical Low-Coherence Reflectometer and Keratometer. Am J Ophthalmol. 2012;153(1):55-61.e2. doi: 10.1016/j.ajo.2011.06.012 pmid: 21907967

19. Bjeloš Rončević M, Bušić M, Čima I, Kuzmanović Elabjer B, Bosnar D, Miletić D. Intraobserver and interobserver repeatability of ocular components measurement in cataract eyes using a new optical low coherence reflectometer. Graefes Arch Clin Exp Ophthalmol. 2011;249(1):83-7. doi: 10.1007/s00417-010-1546-z pmid: 20981435

20. Shen P, Zheng Y, Ding X, Liu B, Congdon N, Morgan I, et al. Biometric measurements in highly myopic eyes. J Cataract Refract Surg. 2013;39(2):180-7. doi: 10.1016/j.jcrs.2012.08.064 pmid: 23228592.

21. Zhao J, Chen Z, Zhou Z, Ding L, Zhou X. Evaluation of the repeatability of the Lenstar and comparison with two other non-contact biometric devices in myopes. Clin Exp Optom. 2013;96(1):92-9. doi: 10.1111/j.1444-0938.2012.00793.x pmid: 22943766.

22. Schulle KL, Berntsen DA. Repeatability of On- and Off-Axis Eye Length Measurements Using the Lenstar. Optom Vis Sci. 2013;90(1):16-22. doi: 10.1097/OPX.0b013e3182780bfd pmid: 23208194

23. Şahin A, Gürsoy H, Başmak H, Yildirim N, Usalp Z, Çolak E. Reproducibility of Ocular Biometry with a New Noncontact Optical Low-Coherence Reflectometer in Children. Eur J Ophthalmol. 2011;21(2):194-8. doi: 10.5301/ejo.2010.1298 pmid: 20853265

24. Kunavisarut P, Poopattanakul P, Intarated C, Pathanapitoon K. Accuracy and reliability of IOL master and A-scan immersion biometry in silicone oil-filled eyes. Eye. 2012;26(10):1344-8. doi: 10.1038/eye.2012.163 pmid: 22878446

25. Roessler GF, Huth JK, Dietlein TS, Dinslage S, Plange N, Walter P, et al. Accuracy and reproducibility of axial length measurement in eyes with silicone oil endotamponade. Br J Ophthalmol. 2009;93(11):1492-4. doi: 10.1136/bjo.2008.152637 pmid: 19628491

26. Parravano M, Oddone F, Sampalmieri M, Gazzaniga D. Reliability of the IOLMaster in axial length evaluation in silicone oil-filled eyes. Eye. 2007;21(7):909-11. doi: 10.1038/sj.eye.6702452 pmid: 16878120

27. Liu GJ, Shen BL, Qiu YJ, Yu Z, Cui HS, Wang HC, Kang WQ, Liu Y. [Clinical results of ultrasound biometry in silicone oil-filled eye]. Zhonghua Yan Ke Za Zhi. 2010 Feb;46(2):113-8. Chinese. pmid: 20388343

28. Wang K, Yuan M-K, Jiang Y-R, Bao Y-Z, Li X-X. Axial length measurements before and after removal of silicone oil: a new method to correct the axial length of silicone-filled eyes for optical biometry. Ophthalmic Physiol Opt. 2009;29(4):449-57. doi: 10.1111/j.1475-1313.2009.00636.x pmid: 19523089

29. Liu R, Li Q. Changes in ocular biometric measurements after vitrectomy with silicone oil tamponade for rhegmatogenous retinal detachment repair. BMC Ophthalmol. 2020;20(1):360. doi: 10.1186/s12886-020-01627-2 pmid: 32878598

30. Kulikov AN, Kokareva EV, Kuznetsov AR. Optical biometry features in silicon oil filled eyes. Ophthalmol J. 2018;11(3):1520. doi: 10.17816/OV11315-20

31. Muthu Krishnan V, Jayalatha K, Vijayakumar C. Correlation of Central Corneal Thickness and Keratometry with Refraction and Axial Length: A Prospective Analytic Study. Cureus. 2019;11(1):e3917. doi: 10.7759/cureus.3917 pmid: 30931188

32. Piasecka K, Bednarski M, Michalewski J, Nawrocki J, Michalewska Z. Optical low-coherence reflectometry in the calculation of intraocular lens power in silicone oil-filled eyes. Klin Oczna. 2016 Sep;117(4):243-248. pmid: 29727110 